\title{
Thiol/disulphide balance in patients with psoriasis
}

\author{
Mustafa Aksoy ${ }^{1}$, Adnan Kirmit ${ }^{2}$ \\ ${ }^{1}$ Department of Dermatology, Faculty of Medicine, Harran University, Şanlıurfa, Turkey \\ ${ }^{2}$ Department of Biochemistry, Faculty of Medicine, Harran University, Şanlıurfa, Turkey
}

Adv Dermatol Allergol 2020; XXXVII (1): 52-55

DOI: https://doi.org/10.5114/ada.2018.77767

\begin{abstract}
Introduction: Psoriasis is a common chronic inflammatory skin disease with unknown etiopathogenesis. Aim: To examine the thiol/disulfide balance in psoriasis patients and to compare the results with a healthy control group. Material and methods: Eighty patients with psoriasis and 80 healthy individuals who were age- and gender-matched with these patients were included in this study. Serum native thiol, disulfide and total thiol levels were measured by a new automated method developed by Erel and Neselioglu. Disulphide/total thiol, disulphide/ native thiol and native thiol/total thiol were calculated.

Results: While there was no statistically significant difference in terms of disulphide levels (SS) between the patient and control groups $(p>0.05)$, there were significant differences in terms of total thiol and native thiol (SH) levels, SS/SH, SS/total thiol and SH/total thiol ratios between the patient and control groups $(p<0.05)$. There was a statistically significant relationship between duration of the disease and the disulfide/native thiol ratio $(p>0.05)$. Conclusions: In recent years, there have been few studies on the role of oxidative stress in the etiopathogenesis of psoriasis. In this study, we investigated in psoriasis patients, thiol/disulfide balance as a new oxidative stress marker. The results were compared with a healthy control group. Our results showed that thiol/disulphide balance shifted towards disulphide in psoriasis patients. This is important as a finding that supports the role of oxidative stress in the pathogenesis of psoriasis.
\end{abstract}

Key words: disulphide, thiol, psoriasis.

\section{Introduction}

Psoriasis is a chronic inflammatory disease of the skin that develops with immune associated mechanisms [1]. The exact pathogenesis of this disease which is characterized by increased keratinocyte proliferation, abnormal keratinocyte differentiation, changes in dermal vascularity, increase in cellular antioxidant activity, and complex existence of dermal and epidermal T cells, monocyte, macrophages and polymorphonuclear leukocytes has not been revealed yet [2].

It has been considered that one of factors that contributes to the initiation of psoriasis or psoriasis to become chronic is reactive oxygen derivatives [3]. It has been proved that the reactive oxygen derivatives formed in keratinocytes, endothelial cells and fibroblasts has chemotactic effects on neutrophils [4]. Neutrophil accumulation in psoriasis lesions causes excessive superoxide development during the phagocytic reaction [5]. Production of excessively reactive oxygen derivatives initiates lipid peroxidation in membranes and causes cell death and the release of arachidonic acid metabolites by activating phospholipase A2. When guanylate cyclase is activated by lipid peroxidation, adenyl cyclase is deactivated. In addition, the CAMP/CGMP ratio decreases and this leads to epidermal hyperproliferation in the psoriasis [6].

A variety of biochemical markers are used to define oxidative stress and inflammation. The dynamic thiol-disulphide balance is one of these markers [7]. Thiols, which constitute a significant amount of the total antioxidants in the body, are compounds that contain sulphur and play an important role in defending against reactive oxygen species [8].

It is known that the plasma total thiol level measurement and the determination of thiol-disulphide balance is a good reflection of excess free radical formation in many diseases [8]. Research on the thiol-disulphide balance has determined that it plays a significant role in the pathogenesis of a few skin diseases such as urticaria [9] and basal cell carcinoma [10]. Psoriasis is one of

Address for correspondence: Assist. Prof. Mustafa Aksoy, Department of Dermatology, Faculty of Medicine, Harran University, 63200 Şanlıurfa, Turkey, phone: +90 5616110142, e-mail: derma63@gmail.com Received: 23.07.2018, accepted: 8.08.2018. 
the diseases in which oxidative stress is a factor in its etiopathogenesis [11]. The thiol/disulphide level measurement can be carried out using a technique developed by Neselioglu and Erel.

\section{Aim}

The aim of this study is to examine the effect of the new oxidative stress indicator, the thiol-disulphide balance in psoriasis patients and to compare the results with a healthy group.

\section{Material and methods}

The study was carried out at a dermatology polyclinic of a state university hospital from 1 May 2017 to 1 August 2017. The local Ethics Committee had issued an approval before the study. Eighty patients with psoriasis and 80 healthy people were included in the study. Psoriasis was diagnosed by clinical findings and skin biopsy. Psoriasis patients undergoing treatment, pregnant and breastfeeding women, and individuals with any systemic or psychiatric disease were not included in the study. The study was carried out after all the participants gave consent and all of the subjects signed the informed consent form. The venous blood samples collected from both the patient and healthy group after at least $8 \mathrm{~h}$ of fasting were centrifuged for $5 \mathrm{~min}$ at $4000 \mathrm{rpm}$ and fractionated and these serums were stored at $-80^{\circ} \mathrm{C}$ in Eppendorf tubes.

The procedures applied in this work were performed in accordance with the Helsinki Declaration.

The thiol/disulphide balance tests were done using a technique developed by Neselioglu and Erel [7]. Initially, the disulphide bonds were reduced to create functional thiol groups together with sodium borohydride. The unused reducing agent sodium borohydride was removed using formaldehyde to prevent the reduction of DTNB (5,5'-dithiobis-(2-nitrobenzoic) acid), all thiol groups including the reduced and native thiol groups were determined after the reaction with DTNB. Half of the difference between total thiols and native thiols was determined by the amount of dynamic disulphide. After the native and total thiols were determined, the disulphide levels, disulphide/total thiol, native thiol/total thiol and disulphide/native thiol ratios were calculated.

\section{Statistical analysis}

The statistical analyses were carried out using IBM SPSS 23.0 (SPSS for Windows, SPSS Inc., Chicago, IL, USA). The Shapiro Wilk test was used as the normality test. Parametric tests were used as the distributions were normal. The differences between the groups were examined using the Student $t$ test and Pearson $\chi^{2}$ test. The limit value for statistically significant differences was 0.05 .

\section{Results}

Of the 80 patients included in the study, 41 were men while 39 were women. The mean age was $30.96 \pm 17.01$. Of the 80 healthy individuals included in the study, 39 were men while 41 were women and the mean age of the healthy group was $26.74 \pm 14.22$. The mean body mass index (BMI) was $24.92 \pm 5.64 \mathrm{~kg} / \mathrm{m}^{2}$ in the patient group and $25.06 \pm 4.92 \mathrm{~kg} / \mathrm{m}^{2}$ in the healthy group. There was no statistically significant difference in age, gender, and BMI between the patient and healthy groups $(p>0.05)$ (Table 1).

Table 1. Comparison of the patients and healthy groups

\begin{tabular}{|c|c|c|c|}
\hline Variable & $\begin{array}{l}\text { Healthy group } \\
\quad(n=80)\end{array}$ & $\begin{array}{l}\text { Patient group } \\
\quad(n=80)\end{array}$ & $P$-value \\
\hline Age, mean \pm SD (min.-max.) [years] & $26.74 \pm 14.22(4-61)$ & $30.96 \pm 17.01(3-70)$ & 0.226 \\
\hline Gender (n/\%): & & & 0.752 \\
\hline Men & $39 / 48.75$ & $41 / 51.25$ & \\
\hline Women & $41 / 51.25$ & $39 / 48.75$ & \\
\hline $\mathrm{BMI}$, mean $\pm \mathrm{SD}\left[\mathrm{kg} / \mathrm{m}^{2}\right]$ & $25.06 \pm 4.92$ & $24.92 \pm 5.64$ & 0.997 \\
\hline Total thiol, mean \pm SD $[\mu \mathrm{mol} / \mathrm{l}]$ & $454.54 \pm 102.04$ & $398.66 \pm 100.41$ & 0.001 \\
\hline Native thiol (SH), $n(\%)[\mu \mathrm{mol} / \mathrm{l}]$ & $90.82(3.64)$ & $88.65(7.34)$ & 0.001 \\
\hline Disulphide (SS), $n(\%)[\mu \mathrm{mol} / \mathrm{l}]$ & $21.55(8.24)$ & $23.30(9.30)$ & 0.128 \\
\hline$\% \mathrm{SS} / \mathrm{SH}, n(\%)$ & $5.05(2.22)$ & $6.40(4.71)$ & 0.009 \\
\hline$\%$ SS/total thiol, $n(\%)$ & $4.58(1.82)$ & $5.67(3.67)$ & 0.006 \\
\hline$\%$ SH/total thiol, $n(\%)$ & $90.82(3.64)$ & $88.65(7.34)$ & 0.006 \\
\hline
\end{tabular}




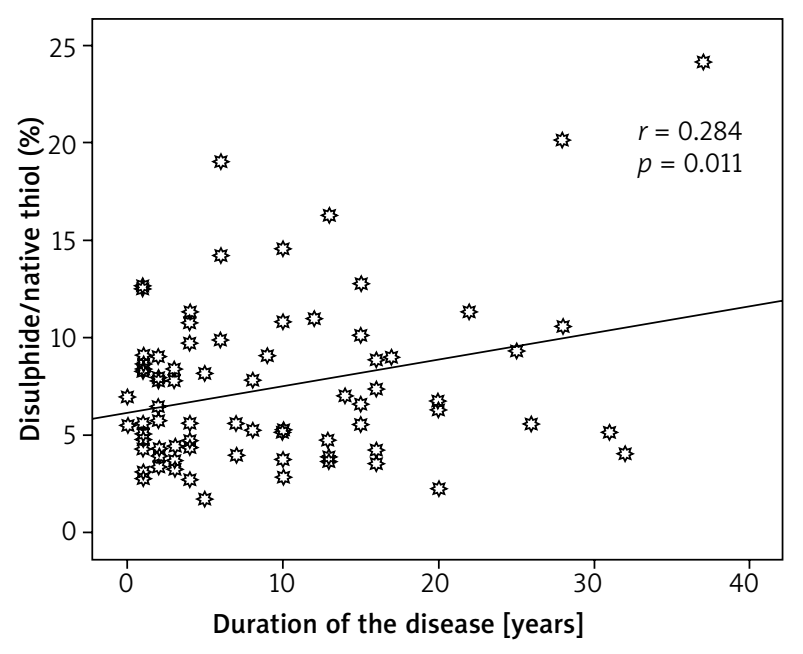

Figure 1. Relationship between the disulphide/native thiol ratio and duration of the disease

While there was no statistically significant difference in terms of disulphide levels (SS) between the patient and healthy groups ( $p=0.128)$, there were significant differences in terms of total thiol and native thiol $(\mathrm{SH})$ levels, SS/SH, SS/total thiol and SH/total thiol ratios between the patient and healthy groups ( $p=0.001, p=$ $0.001, p=0.009, p=0.006$ and $p=0.006$, respectively) (Table 1).

$67.5 \%$ of the patients were early onset ( $\leq 40$ years) and $32.5 \%$ late onset. There was a statistically significant relationship between the disulphide/native thiol ratio and duration of the disease $(p=0.011, r=0.284)$ (Figure 1).

\section{Discussion}

The plasma thiol pool consists fundamentally of albumin thiols, protein thiols, and lower-degree low-molecular-weight thiols. These organic compounds comprise a sulfhydryl group and play a critical role in preventing oxidative stress. The thiol groups of amino acids that contain sulphur such as methionine and cysteine in its protein structure are the primary target of oxygen species. The most common type of thiol found in human cells is the glutathione that creates the optimal redox environment for cell function [7, 12]. Thiol groups create a disulphide bond by oxidation under the impact of reactive oxygen species. The transformation of thiols to disulphides is the earliest indicator of reactive oxygen derivative protein oxidation. Thiol groups that transform into reversible disulphides can be reduced to thiol groups. This allows the protection of thiol-disulphide homeostasis [13]. It is known that the plasma total thiol level measurement and the determination of thiol-disulphide homeostasis is a good reflection of excess free radical formation in many diseases [8].
Free radicals resulting from normal metabolism or pathological processes cause deterioration in the structure and functions of the thiol-related enzymes and the thiol-disulphide balance in the cell environment. A decreased plasma thiol concentration shows an increase in free radical generation. Recently, a technique developed by Erel and Neselioglu has made it possible to obtain information about oxidative stress by determining the total plasma thiol/disulphide ratios [7].

The skin is one of the important targets for oxidative injuries [14]. It is attempted to eliminate toxic effects of free oxygen radicals that are excessively produced on the cellular level by the antioxidant defense system. Oxidative stress that arises when the antioxidant defense system is not sufficient plays a major role in the aging process and evaluation of many diseases $[15,16]$. This clinically causes negative effects such as erythema, edema, wrinkles, photo-aging, inflammation, autoimmune reaction, hypersensitivity, keratinization disorders, and paraneoplastic and neoplastic lesions [17].

The thiol-disulphide balance has been researched in a limited number of dermatoses (urticaria, basal cell carcinoma, atopic dermatitis) until now $[9,10,18]$. Demirsen et al. carried out research on 34 patients with basal cell carcinoma and a group of 30 healthy individuals to determine the thiol-disulphide balance. They observed a statistically significant difference between the disulphide/native thiol and native thiol/total thiol ratios and the serum native thiol and disulphide levels between the patient and healthy groups. This established that the thiol-disulphide balance could be a factor in the pathogenesis of basal cell carcinoma [10]. In another study undertaken by Akbas et al., the thiol/disulphide homeostasis was examined in 53 patients with acute urticaria and 47 healthy individuals, and 57 patients with chronic spontaneous urticaria and 57 healthy individuals. While the thiol/disulphide level did not change in the group with acute urticaria, it had changed in the group with chronic spontaneous urticaria. It also indicated that there was a decrease in the natural and total thiol levels in both acute urticaria and chronic spontaneous urticaria groups, thus suggesting that the activation of urticaria had an impact on the thiol/disulphide levels [9]. In a study carried out by Uysal et al., it has been reported that both oxidative stress and impaired thiol-disulphide balance are associated with childhood atopic dermatitis [18]. Although oxidative stress has been shown to be one of the factors responsible for the pathogenesis of psoriasis in various studies, its role is still unclear. In studies on this subject conflicting results have been reported [14, 19-21].

In the literature, there are few studies performed regarding thiol-disulphide balance in patients with psoriasis. Kilic et al. have run a study on 92 patients with psoriasis and 71 healthy individuals and examined the homeostasis of thiol/disulphide in psoriasis patients and the relationship between this balance and dyslipidemia. 
As a result of this study, lower plasma disulphide levels and higher native thiol levels were detected in patients with psoriasis than healthy individuals [21]. Emre et al. performed a study with 90 patients with psoriasis and 76 healthy individuals and examined the homeostasis of thiol/disulphide. As a result, increased thiol and decreased disulphide levels were detected in patients and it has been stated that this might be responsible from increased keratinocyte proliferation in pathogenesis of psoriasis [22]. A study carried out by Yazici et al. evaluated the thiol levels in patients with psoriasis and found the serum thiol levels in the patient group to be significantly lower than that of the healthy group [11].

\section{Conclusions}

We examined the thiol/disulphide homeostasis in psoriasis patients which is a new oxidative marker and compared the results with the healthy group. This showed that the increase in oxidants in psoriasis patients was higher than in the healthy group. A statistically meaningful relationship between the disulphide/native thiol ratio and duration of the disease indicates that with the longer duration of the disease, the disulphide/native thiol ratio increases and the disulphide/native thiol ratio deteriorates in terms of an increase in oxidants.

With regards to contribution to the literature and support for our results, we consider that more studies should be conducted with more patients.

\section{Conflict of interest}

The authors declare no conflict of interest.

\section{References}

1. Gulekon A, Adisen E. Psoriasis and co-morbidities. Turk Arch Dermatol Venereology 2008; 42: 23-5.

2. Sikar Akturk A, Ozdogan HK, Bayramgurler D, et al. Nitric oxide and malondialdehyde levels in plasma and tissue of psoriasis patients. J Eur Acad Dermatol Venereol 2012; 26: 833-7.

3. Attwa E, Swelam E. Relationship between smoking-induced oxidative stress and the clinical severity of psoriasis. J Eur Acad Dermatol Venereol 2011; 25: 782-7.

4. Raynaud F, Brion DE, Gerbaud P. Oxidative modulation of cyclic AMP-dependent protein kinase in human fibroblasts: possible role in psoriasis. Free Radic Biol Med 1997; 22: 623-32.

5. Turner CP, Toye AM, Jones OTG. Keratinocyte superoxide generation. Free Radic Biol Med 1998; 24: 401-7.

6. Popov I, Lewin G. A deficient function of the antioxidative system of the organism as an aetiopathogenetic factor in psoriasis. Med Hypotheses 1991; 35: 229-36.

7. Erel O, Neselioglu S. A novel and automated assay for thiol/ disulphide homeostasis. Clin Biochem 2014; 47: 326-32.

8. Ozyazıcı S, Karateke F, Turan U, et al. A novel oxidative stress mediator in acute appendicitis: thiol/disulphide homeostasis. Mediators Inflamm 2016; 2016: 6761050.
9. Akbas A, Kilinc F, Sener S, et al. Investigation of thiol/disulphide balance in patients with acute urticaria and chronic spontaneous urticaria. Cutan Ocul Toxicol 2017; 36: 205-10.

10. Demirseren DD, Cicek C, Alisik M, et al. Dynamic thiol/disulphide homeostasis in patients with basal cell carcinoma. Cutan Ocul Toxicol 2017; 36: 278-82.

11. Yazici C, Kose K, Utas S, et al. A novel approach in psoriasis: first usage of known protein oxidation markers to prove oxidative stress. Arch Dermatol Res 2016; 308: 207-12.

12. Bal C, Buyuksekerci M, Koca C, et al. The compromise of dynamic disulphide/thiol homeostasis as a biomarker of oxidative stres in trichloroethylene exposure. Hum Exp Toxicol 2016; 35: 915-20.

13. Ates I, Ozkayar N, Inan B, et al. Dinamic thiol/disulphide homeostasis in patients with newly diagnosed primary hypertension. J Am Soc Hypertens 2016; 10: 159-66.

14. Kadam DP, Suryakar AN, Ankush RD, et al. Role of oxidative stress in various stages of psoriasis. Ind J Clin Biochem 2010; 25: 388-92.

15. Singal PK, Khaper N, Palace V, Kumar D. The role of oxidative stress in the genesis of heart disease. Cardiovasc Res 1998; 40: 426-32.

16. Yagci R, Ozyurt H, Akbas A, et al. Total antioxidant capacity, total oxidant status and dehydroepiandrosterone sulphate levels in Behcet's disease. Ret Vit 2007; 15: 263-6.

17. Karaca S, Guder H. Antioxidant system in dermatology. Turk J Dermatol 2009; 3: 32-9.

18. Uysal P, Avcil S, Neselioglu S, et al. Association of oxidative stress and dynamic thiol-disulphide homeostasis with atopic dermatitis severity and chronicity in children: a prospective study. Clin Exp Dermatol 2018; 43: 124-30.

19. Rocha-Pereira P, Santos-Silva A, Rebelo I, et al. The inflammatory response in mild and in severe psoriasis. Br J Dermatol 2004; 150: 917-28.

20. Severin E, Nave B, Ständer M, et al. Total antioxidative capacity is normal in sera from psoriasis patients despite elevated bilirubin, tocopherol and urate levels. Dermatology 1999; 198: 336-9.

21. Kilic A, Yorulmaz A, Erdogan S, et al. An evaluation of thiol/ disulphide homeostasis in patients with psoriasis. Adv Dermatol Allergol 2017; 34: 464-7.

22. Emre S, Demirseren DD, Alisik M, et al. Dynamic thiol/disulfide homeostasis and effects of smoking on homeostasis parameters in patients with psoriasis. Cutan Ocul Toxicol 2017; 36: 393-6. 